\title{
Summation relation for $U(N)$ Racah coefficients
}

\author{
K. T. Hecht \\ Physics Department, University of Michigan, Ann Arbor, Michigan 48104 \\ (Received 3 April 1974) \\ A summation relation is given for $U(N)$ Racah coefficients which has the form of an orthogonality \\ relation, or a composition of recoupling transformations, except that the summation over column \\ indices (for fixed row indices) is over multiplicity labels only. In the recoupling matrix for $\left[f^{1}\right] \times$ \\ $\left[f^{2}\right] \times\left[f^{3}\right] \rightarrow[f], U(N)$ irreducible representations $\left[f^{2}\right]$ and $\left[f^{3}\right]$ are limited to be elementary, \\ $[11 \ldots 10 \ldots 0] \equiv\left[1^{k}\right]$, or totally symmetric $[k]$, or of the form $\left[k^{N-1}\right]$. Results are tabulated as functions \\ of the axial distances in $[f]$ for $\left[f^{2}\right]=\left[1^{N-1}\right],\left[1^{N-2}\right]$, or $\left[2^{N-1}\right] ;\left[f^{3}\right]=[1]$, $\left[1^{2}\right]$, or [2]; all cases which \\ arise in the evaluation of squares of matrix elements of one- and two-body operators averaged over \\ irreducible representations of $U(N)$.
}

\section{INTRODUCTION}

In recent years the Wigner-Racah calculus for the unitary groups $U(N)$ has been brought to a state of development comparable to that for the angular momentum calculus for $S U(2)$. Biedenharn, Louck, and collaborators, ${ }^{1-6}$ especially, have developed powerful methods which make it possible to calculate all Wigner and Racah coefficients for $U(N)$. For the case of multiplicity free and extremal Wigner couplings, in particular, algebraic formulas for the Wigner coefficients can be read off directly from their diagrammatic pattern calculus. ${ }^{4}$ In more general cases an additional algorithm is needed to extract algebraic or numerical values of the Wigner coefficients from their formalism. In the case of $S U(3)^{2,3}$ this has been translated into a computer program, ${ }^{7,8}$ so that both Wigner and Racah coefficients for $S U(3)$ are now available in complete generality. Biedenharn and Louck advocate the view that there is a canonical structure for the $U(N)$ Wigner-Racah algebra. This eliminates all free choices in the resolution of the multiplicity problem for the general Wigner coupling, so that all $U(N)$ Wigner and Racah coefficients are uniquely defined. For arbitrary $N$, explicit algebraic constructions for Wigner couplings involving the most general multiplicity structure have so far been limited to matrix elements of the Wigner operators transforming as the $U(N)$ irreducible representation [211...10] $\equiv\left[21^{N-2}\right]\{$ equivalent to $[10 \ldots 0-1]$ in $S U(N)\}$. As a by-product of this calculation, Louck and Biedenharn ${ }^{1}$ also give the $U(N)$ Racah coefficients for the recoupling matrix for $[f] \times[11 \ldots 10] \times[10 \ldots 0] \rightarrow[f]$ in elegantly compact form. Although Racah coefficients, being independent of subgroup labels, have a simpler algebraic structure than the Wigner coefficients, general expressions for $U(N)$ Racah coefficients have so far been limited to a few very special cases, usually cases in which the four Wigner couplings in the Racah recoupling transformation are all free of multiplicity such as when two or more of the irreducible representations are totally symmetric (Moshinsky and Chacón ${ }^{9}$ and Ališauskas, Jucys, and Jucys ${ }^{10}$ ). In the applications to physical problems Racah coefficients are often more useful than Wigner coefficients, and it is hoped that the work of Louck and Biedenharn ${ }^{1}$ will be extended to more general cases. Since the algebraic construction for the most general $U(N)$ Racah coefficients is complicated, it may be useful to search for new relations or sum rules for the $U(N)$ Racah coefficients which have no analog for the simpler $S U(2)$ Racah coefficients.
It is the purpose of this note to exhibit such a summation relation. It has the form of the well-known orthogonality relations for the $U(N)$ Racah coefficients, or of a composition of recoupling transformations, except that the summation over column indices (for fixed row index) is over multiplicity labels only, for fixed $U(N)$ irreducible representation label within the column index. This relation is particularly simple if the representations $\left[f^{2}\right]$ and $\left[f^{3}\right]$ in the recoupling matrix for $\left[f^{1}\right] \times\left[f^{2}\right] \times\left[f^{3}\right] \rightarrow[f]$ are either "elementary,"

$[11 \ldots 10 \ldots 0] \equiv\left[1^{k}\right]$, or totally symmetric, $[k 0 \ldots 0]$

$\equiv[k]$, or of the form $[k k \ldots k 0] \equiv\left[k^{N-1}\right]$. In this case the sum is completely independent of the multiplicity structure and can hence be calculated by permutation group techniques. This sum arises naturally ${ }^{11}$ in applications to physical problems, since it is needed in the calculation of squares of matrix elements of operators averaged over the states of irreducible representations of $U(N)$, where these averages are needed in the study of spectroscopic problems using spectral distribution methods. ${ }^{11,12}$ In Sec. 2 the summation relation for $U(N)$ Racah coefficients is related to the matrix element of a projection operator for the symmetric group. Section 3 takes up the calculational tools needed to evaluate this matrix element, including a transformation to nonstandard representations of $S_{n}$. The details of the calculation are exhibited through some illustrative examples in Sec. 4. Finally, results are tabulated for all cases of the recoupling matrix for $[f] \times\left[f^{2}\right] \times\left[f^{3}\right] \rightarrow[f]$, where the $U(N)$ irreducible representations $\left[f^{2}\right]$ are of the form $\left[1^{N-1}\right]$ or $\left[1^{N-2}\right]$ or $\left[2^{N-1}\right]$, and $\left[f^{3}\right]$ is of the form $[1]$ or $\left[1^{2}\right]$ or $[2]$, which are the $U(N)$ ir reducible representations needed to construct all one- and two-body operators through the coupling $\left[f^{2}\right] \times\left[f^{3}\right]$.

\section{THE SUMMATION RELATION}

For present purposes it will be convenient to use a notation for the $U(N)$ Racah coefficient which is a straightforward generalization of that for the angular momentum calculus for $S U(2)$ and give the Racah coefficient in unitary form, the $U$ coefficient, which is given by the recoupling matrix

$$
\begin{aligned}
& U\left(\left[f^{1}\right]\left[f^{2}\right][f]\left[f^{3}\right] ;\left[f^{12}\right] \rho^{12} \rho^{12,3} ;\left[f^{23}\right] \rho^{23} \rho^{1,23}\right) \\
&=\left\langle\left(\left(\left[f^{1}\right] \times\left[f^{2}\right]\right)\left[f^{12}\right] \rho^{12} \times\left[f^{3}\right]\right)[f] \rho^{12,3}\right. \\
& \times\left|\left(\left[f^{1}\right] \times\left(\left[f^{2}\right] \times\left[f^{3}\right]\right)\left[f^{23}\right] \rho^{23}\right)[f] \rho^{1,23}\right\rangle .
\end{aligned}
$$

Here, the irreducible representation labels $\left[f^{s}\right] \equiv\left[f_{i N}^{s}\right]$ are given by the partition numbers $f_{i N}^{s}, i=1, \ldots, N$, which specify the number of squares in the $i$ th row of 
the Young tableau describing the representation $\left[f^{s}\right]$ of $U(N)$. The multiplicity labels $\rho^{s t}$ are needed whenever the Wigner coupling of $\left[f^{s}\right]$ with $\left[f^{t}\right]$ can yield a specific representation $\left[f^{s t}\right]$ with $d$-fold multiplicity, $d>1$. Since the result of this investigation will involve a summation over multiplicity labels only, it is convenient to use a separate symbol for the multiplicity label and avoid the more elegant notation of references, ${ }^{1-6}$ even though the Biedenharn-Louck canonical structure has been adopted for the $U(N)$ Wigner-Racah algebra. Note that the column index for the unitary transformation matrix is specified by both the irreducible representation label $\left[f^{23}\right]$ and the multiplicity labels $\rho^{23}$ and $\rho^{1,23}$; similarly for the row index. In the notation of Louck and Biedenharn, ${ }^{1}$ the above $U$ coefficient is the matrix element of the $U(N)$ Racah invariant operator

$$
\left\{\left(\begin{array}{l}
{\left[f^{23}\right]} \\
\left(\Gamma^{1,23}\right)
\end{array}\right)\left(\begin{array}{l}
\left(\Gamma^{23}\right) \\
{\left[f^{3}\right]} \\
\left(\Gamma^{12,3}\right)
\end{array}\right)\left(\begin{array}{l}
{\left[f^{2}\right]} \\
\left(\Gamma^{12}\right)
\end{array}\right)\right\}
$$

connecting states of irreducible representation $\left[f^{1}\right]$ (on the right) to states $[f]$ (on the left). Here, the labels $\Gamma^{s t}$ include both the multiplicity labels $\rho^{s t}$ and the shift indices, $\Delta_{i}=f_{i N}^{s t}$, which indicate how many of the squares of the Young tableau for $\left[f^{t}\right]$ have been added to the $i$ th row of the tableau for $\left[f^{s}\right]$ to make the tableau for $\left[f^{s t}\right]$.

For recoupling transformations in which the representations $\left[f^{2}\right]$ and $\left[f^{3}\right]$ are restricted to be "elementary" $\left[1^{k}\right]$, totally symmetric $[k]$, or of the form $\left[k^{N-1}\right]$, only the multiplicity label $\rho^{1,23}$ is needed. (The other Wigner couplings are free of multiplicity; whenever a multiplicity label $\rho$ is unnecessary it will be omitted.) In this case the sum

$$
\begin{aligned}
& \sum_{\rho^{1,23}} U\left(\left[f^{1}\right]\left[f^{2}\right][f]\left[f^{3}\right] ;\left[f^{12}\right]_{--} ;\left[f^{23}\right]_{-} \rho^{1,23}\right) \\
& \quad \times U\left(\left[f^{1}\right]\left[f^{2 \prime}\right][f]\left[f^{3 \prime}\right] ;\left[f^{12 \prime}\right]_{--} ;\left[f^{23}\right]_{-} \rho^{1,23}\right)
\end{aligned}
$$

can be evaluated by permutation group techniques. Note that with $\left[f^{2 \prime}\right]=\left[f^{2}\right],\left[f^{3 \prime}\right]=\left[f^{3}\right]$, and a summation over both $\rho^{1,23}$ and $\left[f^{23}\right]$, the above would have become merely one of the orthonormality relations for the $U$ coefficients. The above sum over $\rho$ only, however, is a simple function of the irreducible representation labels $\left[f^{1}\right],\left[f^{23}\right],[f] ;\left[f^{2}\right],\left[f^{3}\right],\left[f^{2 \prime}\right],\left[f^{3 \prime}\right] ;\left[f^{12}\right]$ and $\left[f^{12 \prime}\right]$. It is this function which is to be evaluated in this investigation.

To evaluate the sum of Eq. (3), it is convenient to introduce $n$-particle state vectors $\left|[f] \alpha ; r_{n} r_{n-1} r_{n-2} \cdots r_{1}\right\rangle$ which are simultaneous base vectors for an irreducible representation of $U(N)$ and of the standard YoungYamanouchi representation ${ }^{13}$ of $S_{n}$ (with $n=\sum_{i} f_{i N}$ ), where $\alpha$ stands for a complete set of subgroup labels for $U(N)$ (the Gel'fand labels $f_{i j}$ with $i \leqslant j=1, \ldots, N-1$, could be used, for example $\left.\mathrm{e}^{1-6}\right)$, and where $r_{n} r_{n-1} \cdots r_{1}$ is a standard Yamanouchi symbol. ${ }^{13}$ It will further be useful to transform to a nonstandard representation of $S_{n},{ }^{14,15}$ in which the group of $k$ particles labeled $n$, $n-1, \ldots, n-k+1$ have a definite permutation symmetry, e.g., $\left[1^{k}\right]$ or $[k]$. Such a state vector can then be expanded in terms of $U(N)$ Wigner coefficients

$$
\begin{aligned}
& \left|[f] \alpha ;\left\{r_{n} r_{n-1} \cdots r_{n-k+1}\right\}\left[f_{1}\right]^{r_{n-k}} r_{n-k-1} \cdots r_{1}\right\rangle \\
& \quad=\sum_{\alpha_{1}^{\prime \alpha \alpha}{ }_{1}}\left|\left[f_{1}^{\prime \prime}\right] \alpha_{1}^{\prime \prime}\right\rangle\left|\left[f_{1}\right] \alpha_{1}\right\rangle\left\langle\left[f_{1}^{\prime \prime}\right] \alpha_{1}^{\prime \prime}\left[f_{1}\right] \alpha_{1} \mid[f] \alpha\right\rangle,
\end{aligned}
$$

where the $U(N)$ Wigner coefficient, $\left\langle\left[f_{1}^{\prime \prime}\right] \alpha_{1}^{\prime \prime}\left[f_{1}\right] \alpha_{1} \mid[f] \alpha\right\rangle_{\rho}$, is the matrix element of the Wigner operator \langle\rangle , namely

$$
\left.\left.\left.\langle\underset{(f]}{[\alpha)}| \begin{array}{c}
(\Gamma) \\
{\left[f_{1}\right]} \\
\left(\alpha_{1}\right)
\end{array}\right)\right\rangle \mid \begin{array}{l}
{\left[f_{1}^{\prime \prime}\right]} \\
\left(\alpha_{1}^{\prime \prime}\right)
\end{array}\right)
$$

in the notation of references ${ }^{1-6}$ Note that $\left[f_{1}\right]$ is assumed to be of the form $\left[1^{k}\right],[k]$, or $\left[k^{N-1}\right]$, so that no multiplicity label $\rho$ is needed in the Wigner coefficient of Eq. (4). Note also that the representation $\left[f_{1}^{\prime \prime}\right]$ is determined uniquely by the Yamanouchi symbols $r_{n}, \ldots, r_{n-k+1}$. (The tableau for $\left[f_{1}^{\prime \prime}\right]$ is obtained from the tableau for $[f]$ by removing squares from rows $r_{n}, r_{n-1} \ldots$, and $\left.r_{n-k+1}.\right)$ By repeating this process for a second group of $k^{\prime}$ particles, and expressing the product of $k$-particle and $k^{\prime}$-particle representations $\left[f_{1}\right]$ and $\left[f_{1}^{\prime}\right]$ in terms of coupled $U(N)$ representations $\left[f_{0}\right]$ for the $\left(k+k^{\prime}\right)$-particle state, the $n$-particle state vector can be expanded as

$$
\begin{aligned}
& \left|[f] \alpha ; r\left(k, k^{\prime}\right)_{\left[f_{1} \mid 1 f f_{1}\right]} \ldots\right\rangle \\
& \equiv\left|[f] \alpha ;\left\{r_{n} \cdots r_{n-k+1}\right\}_{\left[f_{1}\right.}\left\{r_{n-k} \cdots r_{n-k-k^{\prime}+1}\right\}_{\left\{f_{1}^{\prime}\right.} r_{n-k-k^{\prime}} \cdots r_{1}\right\rangle \\
& =\sum_{\alpha_{1}^{\prime} \alpha_{1}} \sum_{\alpha^{\prime} \alpha_{1}^{\prime}} \sum_{\left.1 f_{0}\right] \alpha_{0}}\left|\left[f^{\prime}\right] \alpha^{\prime}\right\rangle\left|\left(\left[f_{1}^{\prime}\right] \times\left[f_{1}\right]\right)\left[f_{0}\right] \alpha_{0}\right\rangle \\
& \times\left\langle\left[f^{\prime}\right] \alpha^{\prime}\left[f_{1}^{\prime}\right] \alpha_{1}^{\prime} \mid\left[f_{1}^{\prime \prime}\right] \alpha_{1}^{\prime \prime}\right\rangle\left\langle\left[f_{1}^{\prime \prime}\right] \alpha_{1}^{\prime \prime}\left[f_{1}\right] \alpha_{1} \mid[f] \alpha\right\rangle \\
& \times\left\langle l f_{1}^{\prime}\right] \alpha_{1}^{\prime}\left[f_{1}\right] \alpha_{1}\left|\left[f_{0}\right] \alpha_{0}\right\rangle \text {. }
\end{aligned}
$$

The sums over subgroup labels $\alpha_{1}, \alpha_{1}^{\prime}, \alpha_{1}^{\prime \prime}$ for the product of three $U(N)$ Wigner coefficients can be expressed more simply in terms of $U(N)$ Racah coefficients by

$$
\begin{aligned}
\sum_{\alpha_{1}^{\prime} \alpha_{1}^{\prime} \alpha_{1}} & \left\langle\left[f^{\prime}\right]\left\langle\left[f^{\prime}\right] \alpha^{\prime}\left[f_{1}^{\prime}\right] \alpha_{1}^{\prime} \mid\left[f_{1}^{\prime \prime}\right] \alpha_{1}^{\prime \prime}\right\rangle \alpha_{1}^{\prime} \mid\left[f_{1}^{\prime \prime}\right] \alpha_{1}^{\prime \prime}\right\rangle \\
& \times\left\langle\left[f_{1}^{\prime \prime}\right] \alpha_{1}^{\prime \prime}\left[f_{1}\right] \alpha_{1} \mid[f] \alpha\right\rangle\left\langle\left[f_{1}^{\prime}\right] \alpha_{1}^{\prime}\left[f_{1}\right] \alpha_{1} \mid\left[f_{0}\right] \alpha_{0}\right\rangle \\
& =\sum_{\rho}\left\langle\left[f^{\prime}\right] \alpha^{\prime}\left[f_{0}\right] \alpha_{0} \mid[f] \alpha\right\rangle_{\rho} \\
& \times U\left\langle\left[f^{\prime}\right]\left[f_{1}^{\prime}\right][f]\left[f_{1}\right] ;\left[f_{1}^{\prime \prime}\right] \ldots ;\left[f_{0}\right] \_\rho\right\rangle_{\infty}
\end{aligned}
$$

Since the Wigner coupling $\left[f^{\prime}\right] \times\left[f_{0}\right]$ is in general not free of multiplicity, both $U(N)$ Wigner and Racah coefficients are functions of the multiplicity label $\rho$, and the result involves a sum over this multiplicity label.

To obtain the relation for the sum of Eq. (3), consider the matrix element of a projection operator, $Y^{\left[f_{0}\right]}$,

$$
\left.\left\langle[f] \alpha ; r^{\prime}\left(k_{2}, k_{2}^{\prime}\right)_{\left[f_{2}\right]\left[f_{2}^{\prime}\right]} \cdots\left|Y^{\left[f_{0}\right]}\right|[f] \alpha ; r\left(k_{1}, k_{1}^{\prime}\right)_{\left[f_{1}\right]\left[f_{1}^{\prime} 1^{\prime}\right.} \ldots\right\rangle\right\rangle
$$

with $k_{2}+k_{2}^{\prime}=k_{1}+k_{1}^{\prime}$, where $Y^{\prime} f_{0}^{1}$ is an operator, built from permutation operators for particles labeled $n, n-1, \ldots, n-k_{1}-k_{1}^{\prime}+1$, which projects the representation with Young tableau $\left[f_{0}\right]$ out of an arbitrary $\left(k_{1}\right.$ $\left.+k_{1}^{\prime}\right)$-particle state. By using Eqs. (6) and (7) for both 
state vectors, the matrix element of $Y^{\left(f_{0}{ }^{1}\right.}$ can be expressed as

$$
\begin{aligned}
\left\langle[f] \alpha ; r^{\prime}\left(k_{2}, k_{2}^{\prime}\right)_{\left[f_{2}\right]\left[f_{2}^{\prime}\right]^{\prime}} .\left|Y^{\left[f_{0}\right]}\right|[f] \alpha ; r\left(k_{1}, k_{1}^{\prime}\right)_{\left[f_{1}\right]\left[f_{1}^{\prime}\right]} \ldots\right\rangle \\
=\sum_{\alpha^{\prime} \alpha_{0}} \sum_{\rho \rho^{\prime}}\left\langle\left[f^{\prime}\right] \alpha^{\prime}\left[f_{0}\right] \alpha_{0} \mid[f] \alpha\right\rangle_{\rho} \\
\quad \times\left\langle\left[f^{\prime}\right] \alpha^{\prime}\left[f_{0}\right] \alpha_{0} \mid[f] \alpha\right\rangle_{\rho^{\prime}} \\
\quad \times U\left(\left[f^{\prime}\right]\left[f_{1}^{\prime}\right][f]\left[f_{1}\right] ;\left[f_{1}^{\prime}\right]_{--} ;\left[f_{0}\right]_{-} \rho\right) \\
\quad \times U\left(\left[f^{\prime}\right]\left[f_{2}^{\prime}\right][f]\left[f_{2}\right] ;\left[f_{2}^{\prime \prime}\right] \_;\left[f_{0}\right]_{-} \rho^{\prime}\right),
\end{aligned}
$$

where we have used the property of the projection operator

$$
\left\langle\left[f_{0}^{\prime \prime}\right] \alpha_{0}^{\prime \prime}\left|Y^{\left[f_{0}\right]}\right|\left[f_{0}^{\prime}\right] \alpha_{0}^{\prime}\right\rangle=\delta_{\left[f_{0}\right]\left[f_{0}^{\prime}\right]^{1}} \delta_{\left[f_{0}\right]\left[f_{0}^{\prime \prime}\right]} \delta_{\alpha_{0}^{\prime} \alpha_{0}^{\prime \prime}}
$$

to eliminate sums over $\left[f_{0}\right]$. From the orthonormality of the $U(N)$ Wigner coefficients

$$
\sum_{\alpha^{\prime} \alpha_{0}}\left\langle\left[f^{\prime}\right] \alpha^{\prime}\left[f_{0}\right] \alpha_{0} \mid[f] \alpha\right\rangle_{\rho}\left\langle\left[f^{\prime}\right] \alpha^{\prime}\left[f_{0}\right] \alpha_{0} \mid[f] \alpha\right\rangle_{\rho^{\prime}}=\delta_{\rho \rho^{\prime}}
$$

we then obtain the desired summation relation

$$
\begin{aligned}
& \sum_{\rho} U\left(\left[f^{\prime}\right]\left[f_{1}^{\prime}\right][f]\left[f_{1}\right] ;\left[f_{1}^{\prime \prime}\right]_{-} ;\left[f_{0}\right]_{-} \rho\right) \\
& \quad \times U\left(\left[f^{\prime}\right]\left[f_{2}^{\prime}\right][f]\left[f_{2}\right] ;\left[f_{2}^{\prime \prime}\right]_{-}\left[f_{0}\right]_{-} \rho\right) \\
& =\left\langle[f] \alpha ; r\left(k_{2}, k_{2}^{\prime}\right)_{\left[f_{2}\right]\left[f_{2}^{\prime}\right]} \ldots\left|Y^{\left(f_{0}\right]}\right|[f] \alpha ; r\left(k_{1}, k_{1}^{\prime}\right)_{\left[f_{1}\right]\left[f_{1}^{\prime}\right]} \ldots\right\rangle .
\end{aligned}
$$

Since the projection operator, $Y^{\left[f_{0}\right]}$, serves only to project the representation $\left[f_{0}\right]$ out of an arbitrary $\left(k_{1}\right.$ $\left.+k_{1}^{\prime}\right)$-particle state, its $S_{n}$ subgroup character is completely immaterial. It could be constructed according to the Young-Yamanouchi-Rutherford ${ }^{16}$ prescription; but it is usually much simpler to give it in symmetric or antisymmetric form ${ }^{13}$ for some conveniently labeled tableau (not necessarily a standard labeling), since any normalized linear combination of $Y_{i}^{\left[f_{0}\right]}$ 's with different $S_{n}$ subgroup labels $i$ will serve the purpose.

\section{CALCULATIONAL TOOLS}

The matrix element of $Y^{1 f_{0}{ }^{1}}$ can be related to the basic matrix element of the transpositions $P_{m-1, m}$ in the standard Young-Yamanouchi representation ${ }^{13}$

$$
\begin{aligned}
\left\langle[f] ; \ldots r_{m}\right. & \left.=p, r_{m-1}=q \ldots\left|P_{m-1, m}\right|[f] ; \ldots r_{m}=p, r_{m-1}=q \ldots\right\rangle \\
& =1 / \tau_{p q}, \\
\left\langle[f] ; \ldots r_{m}\right. & \left.=q, r_{m-1}=p \ldots\left|P_{m-1, m}\right|[f] ; \ldots r_{m}=p, r_{m-1}=q \ldots\right\rangle \\
& =\left[1-1 / \tau_{p q}^{2}\right]^{1 / 2},
\end{aligned}
$$

where $\tau_{p q}$ is the "axial distance" between the squares labeled $m$ and $m-1$ in the Young tableau,

$$
\tau_{p q}=f_{p}^{(m)}-f_{q}^{(m-1)}-p+q
$$

and $f_{i}^{(m)}$ is the number of squares in row $i$ of the $m$-particle tableau left, after particles labeled $n, n-1, \ldots$, $m+1$ have been removed from the original $n$-particle tableau of shape $[f]$. If the symbol $r_{m}$ is preceded by $\sigma(p)$ symbols with the label $p$ and the symbol $r_{m-1}$ is preceded by $\sigma(q)$ symbols with the label $q$,

$$
\tau_{p q}=f_{p}-f_{q}-p+q-\sigma(p)+\sigma(q)
$$

where $f_{i}$ now designates the number of squares in the $i$ th row of the $n$-particle tableau, $[f]\left(f_{i} \equiv f_{i N}\right)$. Note that $\tau_{q p}=-\tau_{p q}$, and that $\tau_{p p}=+1$, since $\sigma(q)=\sigma(p)+1$ in this case. Note also that $\tau_{p q}$ can be expressed in terms of differences of "partial hooks,"1-6 $p_{i N}=f_{i N}-i+N$.

The transformation to nonstandard representations of $S_{n}$ is particularly simple if the $k$ particles to be singled out belong to the totally symmetric or antisymmetric representations of $S_{n}$. In this case the transformation coefficients have been given by Horie. ${ }^{15}$ For the totally symmetric case in which the $k$ particles labeled $n-m-1, \ldots, n-m-k$ have been singled out

$$
\begin{aligned}
\mid r_{n} \ldots & \left.r_{n-m}\left\{r_{n-m-1}=a_{1}, \ldots, r_{n-m-k}=a_{k}\right\}_{[k]} r_{n-m-k-1} \ldots r_{1}\right\rangle \\
= & \sum_{p} p\left[\frac{1}{k !_{i<j=1}^{k}}\left(1+\frac{1}{\tau_{a_{i} a_{j}}}\right)\right]^{1 / 2} \\
& \times\left|r_{n} \ldots r_{n-m} a_{1} a_{2} \ldots a_{k} r_{n-m-k-1} \ldots\right\rangle,
\end{aligned}
$$

where the sum is over the $k$ ! permutations $P$ which permute the symbols $a_{1}, \ldots, a_{k}$ in both the state vector and the coefficient. Similarly, for the totally antisymmetric case

$$
\begin{aligned}
\mid r_{n} \ldots & \left.r_{n-m}\left\{r_{n-m-1}=a_{1}, \ldots, r_{n-m-k}=a_{k}\right\}_{\left[1^{k} 1\right.} r_{n-m-k-1} \ldots r_{1}\right\rangle \\
= & \sum_{P} P(-1)^{p+1}\left[\frac{1}{k !} \prod_{k j=1}^{k}\left(1-\frac{1}{\tau_{a_{i} a_{j}}}\right)\right]^{1 / 2} \\
& \times\left|r_{n} \ldots r_{n-m} a_{1} a_{2} \ldots a_{k} r_{n-m-k-1} \ldots\right\rangle
\end{aligned}
$$

with $p=$ even (odd) for even (odd) permutations, $p$.

It will also be useful to build state vectors antisymmetric (or symmetric) in one group of $k$ particles, labeled $n-m-1, \ldots, n-m-k$, from vectors antisymmetric (or symmetric) in the $k-1$ particles $n-m-2, \ldots, n-m-k$ through antisymmetrizers $A$ (or symmetrizers $S$ ), with

$$
A(n-m-1, \ldots, n-m-k)=\frac{1}{k !} \sum_{P} P(-1)^{p}
$$

where the sum runs over the $k !$ permutations $P$ of particles labeled $n-m-1, \ldots, n-m-k$, and $p=$ even (odd) for $P=$ even (odd):

$$
\begin{aligned}
A(n- & m-1, \ldots, n-m-k) \\
& \times\left|r_{n} \ldots r_{n-m} a_{1}\left\{a_{2} a_{3} \ldots a_{k}\right\}_{\left[1,1^{k-1}\right]} r_{n-m-k-1} \ldots r_{1}\right\rangle \\
= & {\left[\frac{1}{k} \prod_{i=2}^{k}\left(1-\frac{1}{\tau_{a_{1} a_{i}}}\right)\right]^{1 / 2} } \\
& \times\left|r_{n} \ldots r_{n-m}\left\{a_{1} a_{2} \ldots a_{k}\right\}_{\left[1^{k} r_{n-m-k-1}\right.} \ldots r_{1}\right\rangle .
\end{aligned}
$$

The inverse transformation gives

$$
\begin{aligned}
\mid \ldots & \left.\left\{a_{1} a_{2} \ldots a_{k}\right\}_{\mathfrak{t}^{k} k_{1}} \ldots\right\rangle \\
= & \sum_{i=1}^{k}(-1)^{i+1}\left[\frac{1}{k} \prod_{\substack{j=1 \\
j \neq i}}^{k}\left(1-\frac{1}{\tau_{a_{i} a_{j}}}\right)\right]^{1 / 2} \\
& \times\left|\ldots a_{i}\left\{a_{1} \ldots a_{i-1} a_{i+1} \ldots a_{k}\right\}_{\left(1^{k-1}\right]} \ldots\right\rangle
\end{aligned}
$$

with analogous expressions for totally symmetric groups. Another useful relation involves the transposition operator which interchanges particles labeled $n-m-1$ and $n-m-k$ in a state vector antisymmetric in particles labeled $n-m-2, \ldots n-m-k$ :

$$
\begin{aligned}
& P_{n-m-1, n-m-k}\left|\ldots a_{1}\left\{a_{2} a_{3} \ldots a_{k}\right\}_{\left[1^{k-1} 1_{1}\right.} \ldots\right\rangle \\
& \quad=\left[\prod_{i=2}^{k}\left(1-\frac{1}{\tau_{a_{1} a_{i}}^{2}}\right)\right]^{1 / 2}\left|\ldots\left\{a_{2} a_{3} \ldots a_{k}\right\}_{\left[1^{k-1}\right]} a_{1} \ldots\right\rangle
\end{aligned}
$$




$$
\begin{aligned}
& +\sum_{i=2}^{k}(-1)^{i} \frac{1}{\tau_{a_{1} a_{i}}}\left[\prod_{\substack{j=2 \\
j \neq i}}^{k}\left(1-\frac{1}{\tau_{a_{1} a_{j}}}\right)\left(1-\frac{1}{\tau_{a_{j} a_{i}}}\right)\right]^{1 / 2} \\
& \times\left|\ldots\left\{a_{1} \ldots a_{i-1} a_{i+1} \ldots a_{k}\right\}_{t^{k-1},} a_{i} \ldots\right\rangle .
\end{aligned}
$$

In the special case $a_{1}=a_{2}$ the above collapses to

$$
\begin{gathered}
P_{n-m-1, n-m-k}\left|\ldots a_{1}\left\{a_{1} a_{3} \ldots a_{k}\right\}_{\left[1^{k-1} 1\right.} \ldots\right\rangle \\
=\left|\ldots\left\{a_{1} a_{3} \ldots a_{k}\right\}_{\left\{1^{k-1} 1\right.} a_{1} \ldots\right\rangle .
\end{gathered}
$$

The transformation to nonstandard representations of $S_{n}$ is complicated in the case where the $k$-particle representations of $S_{n}$ are other than one-dimensional. For a group of $(N-1)_{k}^{n}$ particles of symmetry $[k k \ldots k 0]$ $\equiv\left[k^{N-1}\right]$, however, it is sufficient to construct a single $(N-1) k$-particle state of symmetry $\left[k^{N-1}\right]$, since the properties of $S U(N)$ insure that the Wigner coupling $[f]$ $\times\left[k^{N-1}\right]$ is free of multiplicity. It will be convenient to choose this single state to transform according to the irreducible representation $\left[k^{N-1}\right]$ of $S_{n}$ and to be in normal antisymmetric form in $k$ distinct groups of $(N-1)$ particles each. (We shall use the notation $\left[k^{N-1}\right]_{a}$. Note that this state is a complicated linear combination of the Young-Yamanouchi base vectors for $\left[k^{N-1}\right]$.)

An $(N-1)$-particle state of symmetry $\left[1^{N-1}\right]$ in which the $N-1$ Yamanouchi symbols include the numbers 1 through $N$, with the exception of the specific number $i$, will be denoted by $\{i]_{\left[1^{N-1}\right]}$ :

$$
\left|\ldots\{\bar{i}\}_{\left[1^{N-1}\right.} \ldots\right\rangle \equiv\left|\ldots\{12 \ldots i-1, i+1, \ldots N\}_{\left[1^{N-1}\right.} \ldots\right\rangle
$$

In this notation the state $\left[k^{N-1}\right]_{a}$ can be expanded as

$$
\begin{aligned}
& \left|[f] \ldots\left\{\bar{a}_{1} \bar{a}_{2} \ldots \bar{a}_{k}\right\}_{\left[k^{N-1} 1_{a}\right.} \ldots\right\rangle \\
& =\sum_{p} p\left[\frac{1}{k !} \prod_{k<j=1}^{k}\left(1+\frac{1}{\tau_{\bar{a}_{1} \bar{a}_{j}}}\right)\right]^{1 / 2} \\
& \times\left|[f] \ldots\left\{\bar{a}_{1}\right\}_{\left[1^{N-1} 1\right.}\left\{\bar{a}_{2}\right\}_{\}^{N-1} 1} \ldots\left\{\bar{a}_{k}\right\}_{\left[1^{N-1} 1\right.} \ldots\right\rangle,
\end{aligned}
$$

where the sum is over the $k$ ! permutations $p$ which permute the symbols $\bar{a}_{i}$. The coefficients follow from Eq. (14) and from conjugation properties under $S U(N)$. The state conjugate to $[f]$ transforms according to $S U(N)$ irreducible representation $\left[f^{*}\right]$ with $f_{i}^{*}=f_{1}-f_{N+1-i}$. If the state $[f(\bar{D})]$ is obtained by removing $N-1$ squares from the tableau for $[f]$, one from each $b u t$ the $p$ th row, then the irreducible representation conjugate to $[f(\bar{p})]$ is specified by the tableau $\left[f^{*}(N+1-p)\right]$ which is obtained from $\left[f^{*}\right]$ by removing one square from row $N+1-p$ of $\left[f^{*}\right]$. Thus the function $\tau_{\bar{z}, \bar{a}}$ in Eq. (19) is related to axial distances in $[f]$ in the following way:

$$
\begin{aligned}
\tau_{\bar{a}_{i} \bar{a}_{j}} \equiv \tau_{\bar{a}_{i} \bar{a}_{j}}^{[f]}=\tau_{N+1-a_{i}, N+1-a_{j}}^{\left[f^{*} *_{1}\right.} \\
=f_{N+1-a_{i}}^{*}-f_{N+1-a_{j}}^{*}+\left(N+1-a_{j}\right)-\left(N+1-a_{i}\right) \\
=\left(f_{1}-f_{a_{i}}\right)-\left(f_{1}-f_{a_{j}}\right)+a_{i}-a_{j}=f_{a_{j}}-f_{a_{i}}+a_{i}-a_{j} \\
=\tau_{a_{j} a_{i}} .
\end{aligned}
$$

In particular, therefore,

$$
\begin{aligned}
\left|[f] r_{n} \ldots r_{n-m}\{\bar{p} \bar{q}\}_{\left(2^{N-1} l_{a}\right.} r_{n-m-2 N+1} \ldots r_{1}\right\rangle \\
=\left[\frac{1}{2}\left(1+\frac{1}{\tau_{q p}}\right)\right]^{1 / 2} \mid[f] r_{n} \ldots r_{n-m}\{12 \ldots p-1, p+1, \\
\left.\quad \ldots N\}_{i^{N-1}, 1}\{12 \ldots q-1, q+1, \ldots N\}_{\left[1^{N-1} 1\right.} \ldots\right\rangle \\
+\left[\frac{1}{2}\left(1-\frac{1}{\tau_{q q}}\right)\right]^{1 / 2} \mid[f] r_{n} \ldots r_{n-m}\{12 \ldots q-1, q+1,
\end{aligned}
$$

$$
\left.\ldots N\}_{[1,-1}^{N}\{12 \ldots p-1, p+1, \ldots N\}_{[1 N-1]} \ldots\right\rangle
$$

with

$$
\tau_{a p}=f_{q}-f_{b}-q+p-\sigma(q)+\sigma(p)
$$

for $p \neq q$, where $\sigma(q)$ and $\sigma(p)$ are the number of occurrences of the row numbers $q$ and $p$ in the Yamanouchi symbols $r_{n}, \ldots r_{n-m}$ while $\tau_{q q} \equiv+1$.

In addition to the above results for nonstandard representations of $S_{n}$, the only other calculational tool needed is the value of the simple sum

$$
\sum_{m} \equiv \sum_{i=1}^{m} \prod_{\substack{j=1 \\ j \neq i}}^{m}\left(1-\frac{1}{\tau_{a_{i} a_{j}}}\right)=m \text {. }
$$

The proof follows (see Ref。1): Let $\tau_{a_{j} a_{j}}=\left(x_{i}-x_{j}\right)$. Then $x_{i}$ are real numbers such that $\left(x_{i}-x_{j}\right)^{a_{j}} \neq 0$, for $i \neq j$ and $\Sigma_{m}$ can be expressed as a contour integral in the complex $z$ plane

$$
\Sigma_{m}=-\frac{1}{2 \pi i} \oint \prod_{j=1}^{m}\left(1-\frac{1}{\left(z-x_{j}\right)}\right) d z
$$

where the contour encloses the $m$ simple poles $x_{1}, \ldots, x_{m}$. After expanding the product in the integrand, only the $m$ terms of the form $1 /\left(z-x_{i}\right)$ give a nonzero contribution to the contour integral.

\section{ILLUSTRATIVE EXAMPLES}

The methods used to evaluate the matrix elements of the projection operator $Y^{\left.-f_{0}\right]}$ of Eq. (11) will be illustrated with two examples. Consider first the simplest (and trivial) case: $\left[f_{1}\right]=\left[f_{2}\right]=[1] ;\left[f_{1}^{\prime}\right]=\left[f_{2}^{\prime}\right]=\left[1^{N-1}\right] ;\left[f_{0}\right]$ $=\left[21^{N-2}\right]$. This is a trivial example since the value of the sum of Eq. (3) follows in this case from the orthonormality relation for the $U$ coefficients and the values for the $U$ coefficients with $\left[f_{0}\right]=\left[1^{N}\right] \equiv[0 \ldots 0]$, which are given by simple dimension factors, see Ref. 1 and Eq. (43) below. Nevertheless, the example illustrates the techniques to be used in more complicated cases. In this example it is most convenient to choose the projection operator $Y^{\left[f_{0}\right]}$ in normal antisymmetric form

$$
\begin{aligned}
Y^{\left[21^{N-2}\right)}= & N A(n-1, \ldots, n-N+1) \\
& \times \frac{1}{2}\left(1+P_{n, n-1}\right) A(n-1, \ldots, n-N+1)
\end{aligned}
$$

with a normalization factor $N$ to be determined. In this case

$$
\begin{aligned}
&\left\langle a_{1}\left\{a_{2} a_{3} \ldots a_{N}\right\}_{\left[1^{N-1}\right.} \ldots\left|Y^{\left[21^{N-2}\right.}\right| a_{1}\left\{a_{2} a_{3} \ldots a_{N}\right\}_{\left[_{1}^{N-1}\right]} \ldots\right\rangle \\
&=\frac{1}{2} N\left\{1+\left\langle a_{1}\left\{a_{2} a_{3} \ldots a_{N}\right\}_{\left[1^{N-1}\right.} \ldots\right|\right. \\
&\left.\times P_{n, \pi-1}\left|a_{1}\left\{a_{2} a_{3} \ldots a_{N}\right\}_{[1 N-1} \ldots\right\rangle\right\} \\
&= \frac{1}{2} N\left\{1+\frac{1}{(N-1)} \sum_{i=2}^{N}\left[\frac{1}{\tau_{a_{1} a_{i}}} \prod_{\substack{j=2 \\
j \neq i}}^{N}\left(1-\frac{1}{\tau_{a_{i} a_{j}}}\right)\right]\right\},
\end{aligned}
$$

where Eq. (17) has been used to uncouple the particle numbered $n-1$ from the antisymmetric group, together with the basic matrix element (12). By rewriting $1 / \tau_{a_{1} a_{i}}=\left(1-1 / \tau_{a_{i} a_{1}}\right)-1$, the sum in $\mathrm{Eq}$. (23) becomes

$$
\left[\sum_{i=2}^{N} \prod_{\substack{j=1 \\ j \neq i}}^{N}\left(1-\frac{1}{\tau_{a_{i} a_{j}}}\right)-\sum_{i=2}^{N} \prod_{j=2}^{N}\left(1-\frac{1}{\tau_{a_{i} a_{j}}}\right)\right] \text {. }
$$

By adding and subtracting $\Pi_{j \neq 2}^{N}\left(1-1 / \tau_{a_{1} a_{j}}\right)$ to the first 
term, both sums in (24) can be evaluated with the identity (22) to give

$$
\left[N-\prod_{j=2}^{N}\left(1-\frac{1}{\tau_{a_{1} a_{j}}}\right)-(N-1)\right],
$$

so that

$$
\begin{aligned}
& \left\langle a_{1}\left\{a_{2} \ldots a_{N}\right\}_{\left[1^{N-1}\right.}\left|Y^{\left[21^{N-2}\right.}\right| a_{1}\left\{a_{2} \ldots a_{N}\right\}_{l_{1} N-1} \ldots\right\rangle \\
& \left.\quad=\sum_{\rho} U^{2}\left(\left[f^{\prime}\right]\left[1^{N-1}\right][f][1] ;\left[f^{\prime \prime}\right]=\left[f\left(a_{1}\right)\right]_{--;} ; 21^{N-2}\right]_{-} \rho\right) \\
& \quad=\frac{N}{2} \frac{N}{(N-1)}\left\{1-\frac{1}{N} \prod_{j=2}^{N}\left(1-\frac{1}{\tau_{a_{1} a_{j}}}\right)\right\} .
\end{aligned}
$$

The normalization factor $N$ is most readily determined by choosing the labels $a_{1}$ such that the Racah recoupling transformation collapses to a trivial one-dimensional unitary transformation. In the above example, with $a_{2}=a_{1}=1, a_{3} a_{4} \ldots a_{N}=23 \ldots N-1$, and $[f]=\left[21^{N-2}\right]$, the representation $\left[f^{\prime}\right]$ is the scalar representation $\left[f^{\prime}\right]$ $=[0 \ldots 0]$, so that the square of the single surviving $U$ coefficient in the sum of Eq. (25) has the value unity. In this case, with $a_{2}=a_{1}, \tau_{a_{1}}=+1$, and Eq. (25) is reduced to $1=N N / 2(N-1)$. With this value for $N$, arbitrary $[f]$, and $a_{1} a_{2} \ldots a_{N}=$ any permutation of $12 \ldots N$, Eq. (25) yields

$$
\begin{aligned}
\sum_{\rho} & U^{2}\left([f]\left[1^{N-1}\right][f][1] ;\left[f\left(a_{1}\right)\right]_{-} ;\left[21^{N-2}\right]_{-} \rho\right) \\
= & \left\{1-\frac{1}{N} \prod_{j=2}^{N}\left(1-\frac{1}{\tau_{a_{1} a_{j}}}\right)\right\},
\end{aligned}
$$

where $\left[f\left(a_{1}\right)\right]$ is the representation with a tableau obtained by removing one square from row $a_{1}$ of the tableau for $[f]$. Note that $\left[f^{\prime}\right]=[f(12 \ldots N)]$ (removal of one square from each row of $[f])$, is equivalent to $[f]$ in $S U(N)$.

As a second example consider the case $\left[f_{1}\right]=\left[f_{2}\right]$ $=[2],\left[f_{1}^{\prime}\right]=\left[f_{2}^{\prime}\right]=\left[2^{N-1}\right],\left[f_{0}\right]=\left[21^{N-2}\right]$. With particles numbered $n-2$ through $n-2 N+1$ already prepared with a permutation symmetry $\left[2^{N-1}\right]_{a}$ in the state vectors, it will now be sufficient to choose a projection operator $Y^{\left[f_{0}{ }^{I}\right.}$ of the form

$N A(n-N-1, \ldots, n-2 N+1) A(n-1, \ldots, n-N) S(n, n-2$,

$$
n-N-1) A(n-1, \ldots, n-N) A(n-N-1, \ldots, n-2 N+1)
$$

corresponding to the labeled tableau of Fig. 1. The symmetrizer

$$
S=\frac{1}{2}\left(1+P_{n, n-2}\right) \frac{1}{3}\left(1+P_{n, n-N-1}+P_{n-2, n-N-1}\right) \frac{1}{2}\left(1+P_{n, n-2}\right)
$$

when sandwiched in between the antisymmetrizers of (27) can be written in the form

$$
S=\frac{1}{12}\left(1+P_{n, n-1}\right)\left(1+2 P_{n-1, n-N-1}\right)\left(1+P_{n, n-1}\right) .
$$

The antisymmetrizers, when acting on a state vector of symmetry $\left|\left\{a_{1} a_{2}\right\}_{\{2\}}\left\{\bar{a}_{1} \bar{a}_{2}\right\}_{\left(2^{N-1}\right]} \ldots\right\rangle$ [see Eq. (21)], give

$$
\begin{aligned}
A(n-1 & , \ldots, n-N) A(n-N-1, \ldots, n-2 N+1) \\
& \left|\left\{a_{1} a_{2}\right\}_{[2]}\left\{\bar{a}_{1} \bar{a}_{2}\right\}_{12^{N-1}} \ldots\right\rangle \\
= & \frac{1}{2}\left(1-\frac{1}{\tau_{a_{1} a_{2}}}\right)\left[\frac{1}{N}\left(1-\frac{1}{\left(\tau_{a_{1} a_{2}}+1\right)}\right) \prod_{j=3}^{N}\left(1-\frac{1}{\tau_{a_{1} a_{j}}}\right)\right]^{1 / 2} \\
& \times\left|a_{2}\left\{a_{1} a_{2} \ldots a_{N}\right\}_{\left[1_{1} N\right.}\left\{a_{1} a_{3} \ldots a_{N}\right\}_{1^{N-1} 1} \ldots\right\rangle
\end{aligned}
$$

$$
\begin{aligned}
& -\frac{1}{2}\left(1+\frac{1}{\tau_{a_{1} a_{2}}}\right)\left[\frac{1}{N}\left(1-\frac{1}{\tau_{a_{2} a_{1}}+1}\right) \prod_{j=3}^{N}\left(1-\frac{1}{\tau_{a_{2} a_{j}}}\right)\right]^{1 / 2} \\
& \times \mid a_{1}\left\{a_{1} a_{2} \ldots a_{N}\right\}_{[1}\left\{\left\{a_{2} a_{3} \ldots a_{N}\right\}_{11^{N-1}} \ldots\right\rangle,
\end{aligned}
$$

where Eq. (16) has been used, after $\left|\left\{a_{1} a_{2}\right\}_{121} \ldots\right\rangle$ is expanded through Eq. (14). It is thus sufficient to evaluate the symmetrizer $S$, of Eq. (29), between states of the type $\left|a_{1}\left\{a_{1} a_{2} \ldots a_{N}\right\}_{{ }_{1} N_{1}}\left\{b_{1} b_{2} \ldots b_{N-1}\right\}_{\left[1^{N-1}\right.} \ldots\right\rangle$, where

$$
\begin{aligned}
& \left\langle a_{1}^{\prime}\left\{a_{1}^{\prime} a_{2}^{\prime} \ldots a_{N}^{\prime}\right\}_{{ }_{1} N_{1}}\left\{b_{1}^{\prime} b_{2}^{\prime} \ldots b_{N-1}^{\prime}\right\}_{\left[1^{N-1}{ }_{1}\right.}\right| \\
& \times S\left|a_{1}\left\{a_{1} a_{2} \ldots a_{N}\right\}_{\left[1^{N}\right.}\left\{b_{1} b_{2} \ldots b_{N-1}\right\}_{\left[1^{N-1} 1\right.}\right\rangle \\
& =\frac{1}{6}\left\langle\ldots\left|\left(1+P_{n, n-1}\right)\right| \ldots\right\rangle \\
& +\frac{1}{6}\left(\ldots \mid\left(1+P_{n, n-1}\right) P_{n-1, n-N} P_{n-N, n-N-1} P_{n-1, n-N}(1\right. \\
& \left.+P_{n, n-1}\right)|\ldots\rangle \text {. }
\end{aligned}
$$

Now, using Eqs. (17) and (12), we have

$$
\begin{aligned}
(1+ & \left.P_{n, n-1}\right)\left|a_{1}\left\{a_{1} a_{2} \ldots a_{N}\right\}_{\left[1^{N}\right.} \ldots\right\rangle \\
= & 2\left[\frac{1}{N} \prod_{j=2}^{N}\left(1-\frac{1}{\left(\tau_{a_{1} a_{j}}-1\right)}\right)\right]^{1 / 2}\left|a_{1} a_{1}\left\{\bar{a}_{1}\right\}_{\left[1^{N-1}\right]} \ldots\right\rangle \\
& +\sum_{i=2}^{N}(-1)^{i+1}\left[\frac{2}{N} \frac{\left(\tau_{a_{1} a_{i}}+1\right)}{\left(\tau_{a_{1} a_{i}}-1\right)} \prod_{\substack{j=2 \\
j \neq i}}\left(1-\frac{1}{\tau_{a_{i} a_{j}}}\right)\right]^{1 / 2} \\
& \times\left|\left\{a_{1} a_{i}\right\}_{[2]}\left\{\bar{a}_{i}\right\}_{\left[1^{N-1}\right.} \ldots\right\rangle
\end{aligned}
$$

while, using Eq. (18),

$$
\begin{aligned}
P_{n-1, n-N}(1+ & \left.P_{n, \pi-1}\right)\left|a_{1}\left\{a_{1} a_{2} \ldots a_{N}\right\}_{\left[1_{1} N_{1}\right.} \ldots\right\rangle \\
= & {\left[\frac{1}{N} \prod_{j=2}^{N}\left(1+\frac{1}{\left(\tau_{a_{1} a_{j}}-1\right)}\right)\right]^{1 / 2}\left\{1+\prod_{j=2}^{N}\left(1-\frac{1}{\tau_{a_{1} a_{j}}}\right)\right\} } \\
& \times\left|a_{1}\left\{\bar{a}_{1}\right\}_{\left[1^{N-1}{ }_{1} a_{1} \ldots\right\rangle} \ldots\right\rangle \\
+ & \sum_{i=2}^{N}(-1)^{i+1}\left[\frac{1}{N}\left(1-\frac{1}{\left(\tau_{a_{1} a_{i}}-1\right)}\right) \prod_{\substack{j=2 \\
j \neq i}}^{N}\left(1+\frac{1}{\tau_{a_{i} a_{j}}}\right)\right]^{1 / 2} \\
& \times\left|a_{1}\left\{\bar{a}_{i}\right\}_{\left[1^{N-1}{ }_{1} a_{i}\right.} \ldots\right\rangle \\
+ & \sum_{i=2}^{N}(-1)^{i+1}\left[\frac{1}{N} \prod_{\substack{j=1 \\
j \neq i}}^{N}\left(1-\frac{1}{\tau_{a_{i} a_{j}}}\right)\right]^{1 / 2} \\
& \left.\left.\times \mid a_{i}\left\{\bar{a}_{i}\right\}_{[1}\right\}^{N-1} a_{1} \ldots\right\rangle
\end{aligned}
$$

where repeated use has been made of the identity (22) to simplify the coefficients of the three types of terms.

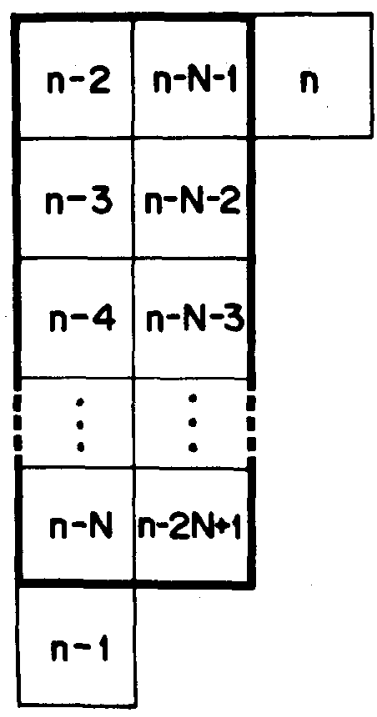


Next, with the shorthand notation $\tau_{a_{1}^{\prime} a_{i}}=\left(\tau_{a_{1} a_{i}}-1\right)=$ $-\tau_{a_{i} a_{1}^{i}}=-\left(\tau_{a_{i} a_{1}}+1\right)$,

$$
\begin{aligned}
& \left\langle a_{1}\left\{a_{1} \ldots a_{N}\right\}_{\mathrm{C}_{1} N_{1}} \ldots\left|\left(1+P_{n, \pi-1}\right)\right| a_{1}\left\{a_{1} \ldots a_{N}\right\}_{\mathrm{C}_{1} N_{1}} \ldots\right\rangle \\
& =\frac{1}{N}\left\{2 \prod_{j=2}^{N}\left(1-\frac{1}{\tau_{a \uparrow a_{j}}}\right)\right. \\
& +2 \sum_{i=2}^{N}\left(1-\frac{1}{\tau_{a_{i} a_{1}^{j}}}\right) \prod_{\substack{j=2 \\
j \neq i}}^{N}\left(1-\frac{1}{\tau_{a_{i} a_{j}}}\right) \\
& \left.-\sum_{\substack{i=2 \\
j \neq 2}}^{N}\left(1-\frac{1}{\tau_{a_{i} a}}\right)\right\} \\
& =\frac{1}{N}\{2 N-(N-1)\}
\end{aligned}
$$

where the sums have again been evaluated with the identity (22). On the other hand,

$\left\langle a_{2}\left\{a_{1} \ldots a_{N}\right\}_{\left[1^{N}\right]} \ldots\left|\left(1+P_{n, n-1}\right)\right| a_{1}\left\{a_{1} \ldots a_{N}\right\}_{\left[1^{N}\right]} \ldots\right\rangle=0$ 。

Finally, from (33), using (17) to uncouple the particle labeled $n-N-1$, we have

$$
\begin{aligned}
& \left\langle a_{1}\left\{a_{1} \ldots a_{N}\right\}_{\left[1^{N}\right]}\left\{b_{1} \ldots b_{N-1}\right\}_{!^{N-1} 1}\right|\left(1+P_{n, n-1}\right) P_{n-1, n N} P_{n-N, n-N-1} \\
& \times P_{n-1, n-N}\left(1+P_{n, n-1}\right) \mid a_{1}\left\{a_{1} \ldots a_{N}\right\}_{\left[1^{N}\right]}\left\{b_{1} \ldots b_{N-1}\right\}_{\left[11^{N-1}\right]} \\
& =\frac{1}{N(N-1)} \sum_{i=1}^{N-1} \prod_{\substack{j=1 \\
j \neq l}}^{N-1}\left(1-\frac{1}{\tau_{b_{i} b_{j}}}\right) F\left(b_{l}\right)
\end{aligned}
$$

with

$$
\begin{aligned}
F\left(b_{1}\right)= & \frac{1}{\left(\tau_{a_{1} b_{l}}-1\right)}\left\{N+2+\prod_{j=2}^{N}\left(1+\frac{1}{\left(\tau_{a_{1} a_{j}}-1\right)}\right)\right\} \\
& +\sum_{i=2}^{N} \frac{1}{\tau_{a_{i} b_{l}}}\left(1+\frac{1}{\left(\tau_{a_{i} a_{l}}-1\right)}\right) \prod_{\substack{j=2 \\
j \neq i_{i}}}^{N}\left(1+\frac{1}{\tau_{a_{i} a_{f}}}\right) \\
= & \frac{(N+2)}{\left(\tau_{a_{1} b_{l}}-1\right)}+1-\left(1-\frac{1}{\left(\tau_{a_{1} b_{l}}-1\right)}\right) \prod_{j=2}^{N}\left(1-\frac{1}{\tau_{a_{j} b_{l}}}\right),
\end{aligned}
$$

where $F\left(b_{t}\right)$ has again been simplified by the use of the identity (22). Finally, the very last product in (37) must always be zero, since $\tau_{a, b}=2$ if $b_{l}=a_{1}$ (in this case $b_{l}$ is preceded by two $a_{1}$ 's in the state vector); and $\tau_{a_{f} b_{l}}$ $=+1$ if $b_{l}=a_{j}$ for any $j \geqslant 2\left(b_{i}\right.$ is now preceded by one $\left.a_{f}\right)$. Hence

$$
F\left(b_{l}\right)=\left\{1+\frac{(N+2)}{\left(\tau_{a_{1} b_{l}}-1\right)}\right\}
$$

and in this form the sum over $l$ in (36) can be performed to give

$$
\begin{aligned}
\sum_{l=1}^{N-1} \prod_{\substack{j=1 \\
j \neq l}}^{N-1}\left(1-\frac{1}{\tau_{b_{l} b_{j}}}\right) F\left(b_{l}\right) & \\
& =\left\{(2 N+1)-(N+2) \prod_{l=1}^{N-1}\left(1-\frac{1}{\left(\tau_{a_{1} b_{l}}-1\right)}\right)\right\} .
\end{aligned}
$$

Combining (31), (34), and (38), we have

$$
\begin{aligned}
& \left\langle a_{1}\left\{a_{1} \ldots a_{N}\right\}_{\left[11^{N}\right.}\left\{b_{1} \ldots b_{N-1}\right\}_{\left[1^{N-1}\right]}\right| \\
& \quad \times S\left|a_{1}\left\{a_{1} \ldots a_{N}\right\}_{\left[1^{N 1}\right]}\left\{b_{1} \ldots b_{N-1}\right\}_{\left[11^{N-1}\right]}\right\rangle
\end{aligned}
$$

$$
=\frac{1}{6} \frac{(N+2)}{(N-1)}\left\{1-\frac{1}{N} \prod_{l=1}^{N-1}\left(1-\frac{1}{\left(\tau_{a_{1} b_{l}}-1\right)}\right)\right\} .
$$

Two cases must be considered: First, if some $b_{f}$ is equal to $a_{1}$, then this $b_{j}$ is preceded by two $a_{1}$ 's, and $\tau_{a_{1} b_{j}}=+2$ for this $j$, so that the product in Eq. (39) is zero. On the other hand, if $b_{i} \neq a_{1}, i=1, \ldots, N-1$, then every $b_{i}$ is preceded by a single $a_{i}$, and $\tau_{a_{1} b_{i}}=\left(\tau_{a_{1} a_{i}}\right.$ $+1)$. In the two cases, Eq. (39) gives

$$
\begin{aligned}
& \left\langle a\{12 \ldots N\}_{\left[1^{N}\right\}}\{\bar{a}\}_{\left[1^{N-1}\right]} \ldots\right| \\
& \times S\left|a\{12 \ldots N\}_{\mathbb{1}^{N} 1}\{\bar{a}\}_{\left.\mathbb{1}^{N-1}\right]} \ldots\right\rangle \\
& \quad=\frac{1}{6} \frac{(N+2)}{(N-1)}\left\{1-\frac{1}{N} \prod_{\substack{i=1 \\
i \neq a}}^{N}\left(1-\frac{1}{\tau_{a i}}\right)\right\}
\end{aligned}
$$

where $a$ is any number from 1 to $N$, while, with $a \neq b$,

$$
\begin{aligned}
& \left\langle a\{12 \ldots N\}_{\left[1^{N}\right]}\{\tilde{b}\}_{\left[1^{N-1}\right]} \ldots\right| \\
& \times s\left|a\{12 \ldots N\}_{\left[1^{N}\right]}\{\bar{b}\}_{\left[11^{N-1}\right.} \ldots\right\rangle \\
& =\frac{1}{6} \frac{(N+2)}{(N-1)} .
\end{aligned}
$$

Using similar techniques, we have

$$
\begin{aligned}
& \left\langle b\{12 \ldots N\}_{\left\{1^{N} 1\right.}\{b\}_{\left\{1^{N-1}\right]}|s| a\{12 \ldots N\}_{\left[1^{N}\right]}\{\bar{a}\}_{\left[_{1} N-1\right.} \ldots\right\rangle \\
& =\frac{(N+2)}{6 N(N-1)}\left[\left(1-\frac{1}{\tau_{a b}^{2}}\right) \prod_{\substack{i=1 \\
i \neq a \neq b}}^{N}\left(1-\frac{1}{\tau_{a i}}\right)\left(1-\frac{1}{\tau_{b i}}\right)\right]^{1 / 2} .
\end{aligned}
$$

Before the final result for the Racah summation relation can be written down, we need to evaluate the normalization factor $N$ of Eq. (27). By choosing $[f]$ $=[32 \ldots 21]$, so that $\left[f^{\prime}\right]=[0 \ldots 0]$, the matrix element of $Y^{11^{N-2_{1}}}$ again has the value unity. Thus

$$
\begin{aligned}
& \left\langle\left[32^{N-2} 1\right] ;\{1 N\}_{[2]}[\bar{N} \bar{N}\}_{\left[2^{N-1}\right]}\right| \\
& \times Y^{\left[21^{N-2}\right]}\left|\left[32^{N-2} 1\right] ;\{1 N\}_{[21}\{\bar{N} \bar{N}\}_{\left(2^{N-1} 1\right.}\right\rangle \\
& =1=N \frac{1}{2}\left(1+\frac{1}{\tau_{1 N}}\right) \frac{1}{N}\left(1-\frac{1}{\left(\tau_{N 1}-1\right)}\right) \prod_{i=2}^{N-1}\left(1-\frac{1}{\tau_{N i}}\right) \\
& \times\left\langle 1\{12 \ldots N\}_{\left[1^{N},\right.}\{\bar{N}\}_{\left[1^{N-1}\right\}}\right| \\
& \times S\left|1\{12 \ldots N\}_{\left[1^{N}\right\}}\{\bar{N}\}_{\left[1^{N-1}\right]}\right\rangle \text {, }
\end{aligned}
$$

with $\tau_{1 N}=N+1=-\tau_{N 1}, \tau_{N i}=-(N-i+1)$, for $i \geqslant 2$, and the matrix element of $S$ given by $(N+2) / 6(N-1)$. Thus

$$
1=\frac{N}{2} \frac{(N+2)}{2 N} \frac{(N+2)}{6(N-1)} .
$$

Finally, with this value of $N$, combining Eqs. (30), (40a), and (40c), the diagonal matrix element of $Y^{\left[21^{N-2}\right]}$ leads to the summation relation

$$
\begin{aligned}
\sum_{\rho} U^{2} & \left([f]\left[2^{N-1}\right][f][2] ;[f(a b)]_{--} ;\left[21^{N-2}\right]_{-} \rho\right) \\
= & \frac{1}{(N+2)}\left\{\frac{\left(\tau_{a b}+1\right)^{2}}{\tau_{a b}\left(\tau_{a b}-1\right)} \prod_{\substack{i \neq a \\
i \neq b}}^{N}\left(1-\frac{1}{\tau_{b i}}\right)\right. \\
& +\frac{\left(\tau_{a b}-1\right)^{2}}{\tau_{a b}\left(\tau_{a b}+1\right)} \prod_{\substack{i \neq a \\
i \neq b}}^{N}\left(1-\frac{1}{\tau_{a i}}\right) \\
& \left.-\frac{4}{N} \prod_{\substack{i \neq a \\
i \neq b}}^{N}\left(1-\frac{1}{\tau_{a i}}\right)\left(1-\frac{1}{\tau_{b i}}\right)\right\}
\end{aligned}
$$

where $\left[f^{\prime \prime}\right]=[f(a b)]$ is the representation with a tableau obtained by removing one square each from row $a$ 
and $b$ of the tableau for $[f]$. Note that in this particular example $\left[f^{\prime}\right]$ is again equivalent to $[f]$ in $S U(N)$, since $\left[f^{\prime}\right]$ is obtained from $[f]$ by removing two squares from every row of the tableau for $[f]$.

\section{RESULTS}

The summation relation (11) arises naturally in applications to physical problems ${ }^{11}$ through the squares of matrix elements of operators, averaged over the states of an irreducible representation of $S U(N)$. [ The reader is directed to Ref. 11 which shows in detail how the $S U(N)$ Racah sum, Eq. (11), arises in averaging the square of an operator or the product of two operators over the states of an irreducible representation of $S U(N)$. Reference 11 also gives a number of applications to nuclear spectroscopic problems using the methods of spectral distributions.] The operators of greatest interest are one- and two-body operators for which the representations $\left[f_{1}^{\prime}\right]$ and $\left[f_{1}\right]$ in the Racah recoupling matrix for $\left[f^{\prime}\right] \times\left[f_{1}^{\prime}\right] \times\left[f_{1}\right] \rightarrow[f]$ are of the type $\left[f_{1}^{\prime}\right]$ $=\left[1^{N-1}\right],\left[1^{N-2}\right]$, or $\left[2^{N-1}\right] ;\left[f_{1}\right]=[1],\left[1^{2}\right]$, or $[2]$. Since $\left[f_{1}^{\prime}\right]$ is then a representation conjugate to a one- or twoparticle representation, it will be natural to denote it by $\left[f_{1}^{\prime *}\right]$, with $\left[1^{N-2}\right]=\left[1^{2 *}\right]$, for example. To eliminate trivial dimensional factors, it will also be useful to tabulate results in the form

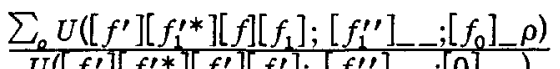

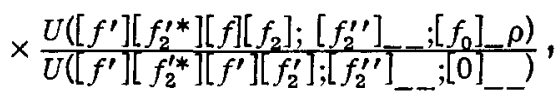

where the $U$ coefficients in the denominator, with $\left[f_{0}\right]$ $=[0 \ldots 0] \equiv[0]$, equivalent to $\left[1^{N}\right]$ or $\left[2^{N}\right]$ in $S U(N)$ are given by trivial dimensional factors. By evaluating these coefficients by the techniques outlined in Secs. 3 and 4 , the summation relation in the form of $\mathrm{Eq}$. (11') also becomes as much as possible independent of specific phase conventions for the $U(N)$ Wigner-Racah algebra. It will be useful to express the $U$ coefficients with $\left[f_{0}\right]=[0]$ as functions of the axial distances in $[f]$. The needed coefficients are

$$
\begin{aligned}
& U\left([f]\left[1^{N-1}\right][f][1] ;[f(a)] ;[0]\right) \\
& =(-1)^{a+1}\left[\frac{1}{N} \prod_{i \neq a}^{N}\left(1-\frac{1}{\tau_{a i}}\right)\right]^{1 / 2}, \\
& U\left([f]\left[1^{N-2}\right][f]\left[1^{2}\right] ;[f(a b)] ;[0]\right) \\
& =(-1)^{a+b}\left[\frac{2}{N(N-1)} \prod_{\substack{i \neq a \\
i \neq b}}^{N}\left(1-\frac{1}{\tau_{a i}}\right)\left(1-\frac{1}{\tau_{b i}}\right)\right]^{1 / 2}, \\
& U\left([f]\left[2^{N-1}\right][f][2] ;[f(a a)] ;[0]\right) \\
& =\left[\frac{2}{N(N+1)} \mathrm{f}_{i \neq a}\left(1-\frac{2}{\tau_{a i}}\right)\right]^{1 / 2}, \\
& U\left([f]\left[2^{N-1}\right][f][2] ;[f(a b)] ;[0]\right) \\
& =(-1)^{a+b}\left[\frac{2}{N(N+1)} \prod_{\substack{i \neq a \\
i \neq b}}^{N}\left(1-\frac{1}{\tau_{a i}}\right)\left(1-\frac{1}{\tau_{b i}}\right)\right]^{1 / 2},
\end{aligned}
$$

where $[f(a b)]$ is again specified by a tableau obtained by removing one square each from row $a$ and $b$ of the tableau for $[f]$, with $a$ and $b$ any of the numbers from 1 to $N$. The possible irreducible representations $\left[f_{0}\right]$ for one- and two-body operators, can be read off from the direct products $\left[f_{1}^{\prime *}\right] \times\left[f_{1}\right]=\Sigma\left[f_{0}\right]$ :

$$
\begin{aligned}
& \text { 1. }\left[1^{N-1}\right] \times[1]=[0]+\left[21^{N-2}\right], \\
& \text { 2. }\left[1^{N-2}\right] \times\left[1^{2}\right]=[0]+\left[21^{N-2}\right]+\left[2^{2} 1^{N-4}\right], \\
& \text { 3. }\left[2^{N-1}\right] \times[2]=[0]+\left[21^{N-2}\right]+\left[42^{N-2}\right], \\
& \text { 4. }\left[2^{N-1}\right] \times\left[1^{2}\right]=\left[21^{N-2}\right]+\left[3^{2} 2^{N-3}\right], \\
& \text { 5. }\left[1^{N-2}\right] \times[2]=\left[21^{N-2}\right]+\left[31^{N-3}\right] .
\end{aligned}
$$

Representations such as $\left[f^{\prime}\right]=\left[f\left(a_{1}^{3} a_{2} a_{3} \ldots a_{N=2}\right)\right]$ or $\left[f^{\top}\right]=\left[f\left(a_{1}^{4} a_{2}^{2} a_{3}^{2} \ldots a_{N-1}^{2}\right)\right]$ can be reached from $[f]$ by only a single operator $Y^{\left[f_{0}\right]}$. E.g., $\left[f_{0}\right]=\left[31^{N-1}\right]$ if $\left[f_{1}^{\prime *}\right]\left[f_{1}\right]$ $=\left[1^{N-2}\right][2]$, or $\left[f_{0}\right]=\left[42^{N-2}\right]$ if $\left[f_{1}^{\prime *}\right]\left[f_{1}\right]=\left[2^{N-1}\right][2]$, for $\left[f^{\prime}\right]=\left[f\left(a_{1}^{3} a_{2} a_{3} \ldots a_{N-2}\right)\right] .\left\{\left[f^{\prime}\right]\right.$ is the $S U(N)$ representation described by the tableau obtained by removing three squares from row $a_{1}$ and one each from rows $a_{2}$ through $a_{N=2}$ from the tableau for $[f]$, where the $a_{i}$ are any of the numbers from 1 through $N$; note that $\left[f\left(a_{1}^{3} a_{2} a_{3} \ldots a_{N-2}\right)\right]$ is equivalent to $\left[f\left(a_{1}^{4} a_{2}^{2} a_{3}^{2} a_{4}^{2} \ldots a_{N-2}^{2} a_{N-1} a_{N}\right)\right.$ in $S U(N)$. \} For such representations the summation relation (11) is trivial, since the Racah recoupling matrix becomes one-dimensional. The only nontrivial cases therefore involve irreducible representations:

$$
\text { A. } \begin{aligned}
{\left[f^{\prime}\right] } & =\left[f\left(a_{1}^{2} a_{2} \ldots a_{N-1}\right)\right] \\
& \equiv\left\{\left[f\left(a_{1}^{3} a_{2}^{2} \ldots a_{N-1}^{2} a_{N}\right)\right] \text { in } S U(N)\right\}
\end{aligned}
$$

and

$$
\text { B. } \begin{aligned}
{\left[f^{\prime}\right]=} & {[f] } \\
& \equiv\left\{\left[f\left(a_{1} a_{2} \ldots a_{N}\right)\right] \equiv\left[f\left(a_{1}^{2} a_{2}^{2} \ldots a_{N}^{2}\right)\right] \text { in } S U(N)\right\} .
\end{aligned}
$$

Results for case A have been tabulated in Ref. 11. Results for case $B$ (diagonal matrix elements) are collected in Table I. The summation relation, in the form (11'), with $\left[f_{1}^{* *}\right]\left[f_{1}\right]=\left[f_{2}^{\prime *}\right]\left[f_{2}\right]$ for the five types of one- and two-body operators enumerated in Eq. (44) are tabulated as cases $1-9$. With $\left[f_{0}\right]=\left[21^{-2}\right]$, it is possible to have $\left[f_{1}^{\prime *}\right]\left[f_{1}\right] \neq\left[f_{2}^{\prime *}\right]\left[f_{2}\right]$. There are ten such possibilities for one- and two-body operators which are tabulated as cases $10-19$ in Table $I$.

TABLE I. The sums

$$
\sum_{\rho} \frac{U\left([f]\left[f_{1}^{\prime *}\right][f]\left[f_{1}\right] ;\left[f_{1}^{\prime \prime}\right]\left[f_{0}\right] \rho\right)}{U\left([f]\left[f_{1}^{\prime *}\right][f]\left[f_{1}^{\prime}\right] ;\left[f_{1}^{\prime \prime}\right][0]\right)} \times \frac{U\left([f]\left[f_{2}^{\prime *}\right][f]\left[f_{2}^{\prime}\right] ;\left[f_{2}^{\prime \prime}\right]\left[f_{0}\right] \rho\right)}{U\left([f]\left[f_{2}^{\prime *}\right][f]\left[f_{2}^{\prime}\right] ;\left[f_{2}^{\prime \prime}\right][0]\right)}
$$

where

$$
\Pi_{a}=\prod_{\substack{i \neq a \\ i \neq b}}\left(1-1 / \tau_{a i}\right), \quad \Pi_{b}=\prod_{\substack{i \neq a \\ i \neq b}}\left(1-1 / \tau_{b i}\right)
$$

\begin{tabular}{ll}
\hline \hline$\left[f_{1}^{\prime}\right]\left[f_{2}^{\prime \prime}\right]$ & 1. $\left[f_{1}^{*}\right]\left[f_{1}\right]=\left[f_{2}^{\prime *}\right]\left[f_{2}\right]=\left[1^{N-1}\right][1] ;\left[f_{0}\right]=\left[21^{N-2}\right]$ \\
\hline$[f(a)][f(a)]$ & $\left\{N \frac{\tau_{a b}}{\left(\tau_{a b}-1\right) \Pi_{a}}-1\right\}$ \\
{$[f(a)][f(b)]$} & -1 \\
\hline$\left[f_{1}^{\prime \prime}\right]\left[f_{2}^{\prime \prime}\right]$ & $2 .\left[f_{1}^{\prime *}\right]\left[f_{1}\right]=\left[f_{2}^{\prime *}\right]\left[f_{2}\right]=\left[1^{N-2}\right]\left[1^{2}\right] ;\left[f_{0}\right]=\left[21^{N-2}\right]$ \\
\hline$[f(a b)][f(a b)]$ & $\frac{N(N-1)}{2(N-2)}\left\{\left(1+\frac{1}{\tau_{a b}}\right) \frac{1}{\Pi_{b}}+\left(1-\frac{1}{\tau_{a b}}\right) \frac{1}{\Pi_{a}}-\frac{4}{N}\right\}$ \\
{$[f(a c)][f(a b)]$} & $\frac{N(N-1)}{2(N-2)}\left\{\left(1-\frac{1}{\tau_{a c}}\right) \frac{1}{\Pi_{a}}-\frac{4}{N}\right\}$ \\
{$[f(c d)][f(a b)]$} & $-\frac{2(N-1)}{(N-2)}$ \\
\hline
\end{tabular}

$\left[f_{1}^{\prime \prime}\right]\left[f_{2}^{\prime}\right] \quad$ 3. $\left[f_{1}^{*}\right]\left[f_{1}\right]=\left[f_{2}^{*}\right]\left[f_{2}\right]=\left[1^{N-2}\right]\left[1^{2}\right] ;\left[f_{0}\right]=\left[2^{2} 1^{N-4}\right]$


Table I (continued)

\begin{tabular}{ll}
{$[f(a b)][f(a b)]$} & $\frac{N}{(N-2)}\left\{1+\frac{(N-1)(N-2)}{2 \Pi_{a} \Pi_{b}}-\frac{(N-1)}{2}\right.$ \\
& $\left.\times\left[\left(1+\frac{1}{\tau_{a b}}\right) \frac{1}{\Pi_{b}}+\left(1-\frac{1}{\tau_{a b}}\right) \frac{1}{\Pi_{a}}\right]\right\}$ \\
{$[f(a c)][f(a b)]$} & $\frac{N(N-1)}{2(N-2)}\left\{\frac{2}{(N-1)}-\frac{\left(\tau_{a c}-1\right)}{\tau_{a c} \Pi_{a}}\right\}$ \\
$\frac{[f(c d)][f(a b)]}{\left[f_{1}^{\prime}\right]\left[f_{2}^{\prime}\right]}$ & $\frac{N}{(N-2)}$ \\
\hline$[f(a b)][f(a b)]$ & $4 .\left[f_{1}^{\prime *}\right]\left[f_{1}\right]=\left[f_{2}^{\prime *}\right]\left[f_{2}\right]=\left[2^{N-1}\right][2] ;\left[f_{0}\right]=\left[21^{N-2}\right]$ \\
{$[f(a a)][f(a a)]$} & $\frac{N(N+1)}{2(N+2)}\left\{\frac{\left(\tau_{a b}-1\right)^{2}}{\tau_{a b}\left(\tau_{a b}+1\right)} \frac{1}{\Pi_{b}}+\frac{\left(\tau_{a b}+1\right)^{2}}{\tau_{a b}\left(\tau_{a b}-1\right)} \frac{1}{\Pi_{a}}-\frac{4}{N}\right\}$ \\
{$[f(a a)]$ or $[f(b b)]$} & $\frac{2 N(N+1)}{(N+2)}\left\{\frac{\tau_{a b}}{\left(\tau_{a b}-1\right)} \frac{1}{\Pi_{a}}-\frac{1}{N}\right\}$ \\
{$[f(a c)]$} & $-\frac{2(N+1)}{(N+2)}$ \\
{$[f(a b)][f(a c)]$} & $\frac{N(N+1)}{(N+2)}\left\{\frac{\left(\tau_{a b}+1\right)}{\left(\tau_{a b}-1\right)} \frac{1}{\Pi_{a}}-\frac{2}{N}\right\}$ \\
{$[f(a b)][f(c d)]$} & $\frac{N(N+1)}{2(N+2)}\left\{\frac{\left(\tau_{a b}+1\right)}{\left(\tau_{a b}-1\right)}\left(1+\frac{1}{\tau_{a c}}\right) \frac{1}{\Pi_{a}}-\frac{4}{N}\right\}$ \\
$\frac{[f(N)]}{(N+2)}$ & $-\frac{2(N+1)}{(N+2)}$
\end{tabular}

\begin{tabular}{|c|c|}
\hline$\left[f_{1}^{\prime \prime}\right]\left[f_{2}^{\prime \prime}\right]$ & 5. $\left[f_{1}^{\prime}\right]\left[f_{1}\right]=\left[f_{2}^{\prime}\right]\left[f_{2}\right]=\left[2^{N-1}\right][2] ;\left[f_{0}\right]=\left[42^{N-2}\right]$ \\
\hline$[f(a b)][f(a b)]$ & $\frac{N(N+1)}{2}\left\{\frac{1}{\Pi_{a} \Pi_{b}}+\frac{2}{(N+1)(N+2)}\right.$ \\
\hline & $\left.-\frac{1}{(N+2)}\left[\frac{\left(\tau_{a b}-1\right)^{2}}{\tau_{a b}\left(\tau_{a b}+1\right)} \frac{1}{\Pi_{b}}+\frac{\left(\tau_{a b}+1\right)^{2}}{\tau_{a b}\left(\tau_{a b}-1\right)} \frac{1}{\Pi_{b}}\right]\right\}$ \\
\hline$[f(a a)][f(a a)]$ & $\frac{N}{(N+2)}\left\{1-2(N+1) \frac{\tau_{a b}}{\left(\tau_{a b}-1\right)} \frac{1}{\Pi_{a}}\right.$ \\
\hline & $\left.+\frac{(N+1)(N+2)}{2} \frac{\tau_{a b}}{\left(\tau_{a b}-2\right)} \prod_{\substack{i \neq a \\
i \neq b}} \frac{\tau_{a i}}{\left(\tau_{a i}-2\right)}\right\}$ \\
\hline$[f(a a)]$ or $\left[\begin{array}{l}{[f(b b)]} \\
{[f(b c)]}\end{array}\right.$ & $\frac{N}{(N+2)}$ \\
\hline$[f(a a)][f(a b)]$ & $\frac{N}{(N+2)}\left\{1-\frac{(N+1)\left(\tau_{a b}+1\right)}{\prod_{a}\left(\tau_{a b}-1\right)}\right\}$ \\
\hline$[f(a b)][f(a c)]$ & $\frac{N}{(N+2)}\left\{1-\frac{(N+1)}{2} \frac{\left(\tau_{a b}+1\right)\left(\tau_{a c}+1\right)}{\left(\tau_{a b}-1\right) \tau_{a c} \Pi_{a}}\right\}$ \\
\hline$[f(a b)][f(c d)]$ & $\frac{N}{(N+2)}$ \\
\hline$\left[f_{1}^{\prime}\right]\left[f_{2}^{\prime \prime}\right]$ & 6. $\left[f_{1}^{*}\right]\left[f_{1}\right]=\left[f_{2}^{*}\right]\left[f_{2}\right]=\left[2^{N-1}\right]\left[1^{2}\right] ;\left[f_{0}\right]=\left[21^{N-2}\right]$ \\
\hline$[f(a b)][f(a b)]$ & $\frac{(N+1)}{2}\left\{\left(1+\frac{1}{\tau_{a b}}\right) \frac{1}{\Pi_{a}}+\left(1-\frac{1}{\tau_{a b}}\right) \frac{1}{\Pi_{b}}\right\}$ \\
\hline$[f(a c)][f(a b)]$ & $\frac{(N+1)}{2}\left[\frac{\left(\tau_{a b}+1\right)}{\left(\tau_{a b}-1\right)}\left(1-\frac{1}{\tau_{a c}^{2}}\right)\right]^{1 / 2} \frac{1}{\Pi_{a}}$ \\
\hline$[f(c d)][f(a b)]$ & \\
\hline
\end{tabular}

\begin{tabular}{lc}
\hline$\left[f_{1}^{\prime \prime}\right]\left[f_{2}^{\prime \prime}\right]$ & 7. $\left[f_{1}^{\prime *}\right]\left[f_{1}\right]=\left[f_{2}^{\prime *}\right]\left[f_{2}\right]=\left[2^{N-1}\right]\left[1^{2}\right] ;\left[f_{0}\right]=\left[3^{2} 2^{N-3}\right]$ \\
\hline$[f(a b)][f(a b)]$ & $\frac{N(N+1)}{2 \Pi_{a} \Pi_{b}}\left\{1-\frac{1}{N}\left[\left(1-\frac{1}{\tau_{a b}}\right) \Pi_{a}+\left(1+\frac{1}{\tau_{a b}}\right) \Pi_{b}\right]\right\}$ \\
{$[f(a c)][f(a b)]$} & $-\frac{(N+1)}{2}\left[\frac{\left(\tau_{a b}+1\right)}{\left(\tau_{a b}-1\right)}\left(1-\frac{1}{\tau_{a c}^{2}}\right)\right]^{1 / 2} \frac{1}{\Pi_{a}}$ \\
{$[f(c a)][f(a b)]$} & 0
\end{tabular}

Table I (continued)

\begin{tabular}{|c|c|}
\hline$\left[f_{1}^{\prime \prime}\right]\left[f_{2}^{\prime \prime}\right]$ & 8. $\left[f_{1}^{*}\right]\left[f_{1}\right]=\left[f_{2}^{*}\right]\left[f_{2}\right]=\left[1^{N-2}\right][2] ;\left[f_{0}\right]=\left[21^{N-2}\right]$ \\
\hline$[f(a b)][f(a b)]$ & $\frac{(N-1)}{2}\left\{\left(1+\frac{1}{\tau_{a b}}\right) \frac{1}{\Pi_{a}}+\left(1-\frac{1}{\tau_{a b}}\right) \frac{1}{\Pi_{b}}\right\}$ \\
\hline$[f(a c)][f(a b)]$ & $\frac{(N-1)}{2}\left[\frac{\left(\tau_{a b}+1\right)}{\left(\tau_{a b}-1\right)}\left(1-\frac{1}{\tau_{a c}^{2}}\right)\right]^{1 / 2} \frac{1}{\Pi_{a}}$ \\
\hline$[f(c d)][f(a b)]$ & 0 \\
\hline$\left[f_{1}^{\prime \prime}\right]\left[f_{2}^{\prime \prime}\right]$ & 9. $\left[f_{1}^{\prime *}\right]\left[f_{1}\right]=\left[f_{2}^{\prime *}\right]\left[f_{2}\right]=\left[1^{N-2}\right][2] ;\left[f_{0}\right]=\left[31^{N-3}\right]$ \\
\hline$[f(a b)][f(a b)]$ & $\frac{N(N-1)}{2 \Pi_{a} \Pi_{a}}\left\{1-\frac{1}{N}\left[\left(1-\frac{1}{\tau_{a b}}\right) \Pi_{a}+\left(1+\frac{1}{\tau_{a b}}\right) \Pi_{b}\right]\right\}$ \\
\hline$[f(a c)][f(a b)]$ & $-\frac{(N-1)}{2}\left[\frac{\left(\tau_{a b}+1\right)}{\left(\tau_{a b}-1\right)}\left(1-\frac{1}{\tau_{a c}^{2}}\right)\right]^{1 / 2} \frac{1}{\Pi_{a}}$ \\
\hline$[f(c d)][f(a b)]$ & 0 \\
\hline
\end{tabular}

\begin{tabular}{lc}
\hline$\left[f_{1}^{\prime \prime}\right]\left[f_{2}^{\prime \prime}\right]$ & $\begin{array}{r}10 . \\
\left.f_{1}^{\prime *}\right]\left[f_{1}\right]=\left[1^{N-1}\right][1] ;\left[f_{2}^{*} *\right]\left[f_{2}\right]=\left[1^{N-2}\right]\left[1^{2}\right] ; \\
{\left[f_{0}\right]=\left[21^{N-2}\right]}\end{array}$ \\
\hline$[f(a)][f(a b)]$ & $N\left[\frac{N-1}{2(N-2)}\right]^{1 / 2}\left\{\frac{1}{\Pi_{a}}-\frac{2}{N}\right\}$ \\
{$[f(a)][f(b c)]$} & $-\left[\frac{2(N-1)}{(N-2)}\right]^{1 / 2}$
\end{tabular}

$\left[f_{1}^{\prime \prime}\right]\left[f_{2}^{\prime \prime}\right] \quad$ 11. $\left[f_{1}^{\prime *}\right]\left[f_{1}\right]=\left[1^{N-1}\right][1] ;\left[f_{2}^{\prime *}\right]\left[f_{2}\right]=\left[2^{N-1}\right][2] ;$

\begin{tabular}{|c|c|}
\hline & {$\left[f_{0}\right]=\left[21^{N-2}\right]$} \\
\hline$[f(a)][f(a a)]$ & $-\left[\frac{2(N+1)}{(N+2)}\right]^{1 / 2}\left\{\frac{N \tau_{a b}}{\left(\tau_{a b}-1\right) \Pi_{a}}-1\right\}$ \\
\hline$[f(a)][f(a b)]$ & $-\left[\frac{N+1}{2(N+2)}\right]^{1 / 2}\left\{\frac{N\left(\tau_{a b}+1\right)}{\left(\tau_{a b}-1\right) \Pi_{a}}-2\right\}$ \\
\hline$[f(a)]$ or $\begin{array}{l}{[f(b b)]} \\
{[f(b c)]}\end{array}$ & {$\left[\frac{2(N+1)}{(N+2)}\right]^{1 / 2}$} \\
\hline$\left[f_{1}^{\prime \prime}\right]\left[f_{2}^{\prime \prime}\right]$ & 12. $\begin{aligned} {\left[f_{1}^{\prime *}\right]\left[f_{1}\right] } & =\left[1^{N-1}\right][1] ;\left[f_{2}^{\prime *}\right]\left[f_{2}\right] \\
& =\left[2^{N-1}\right]\left[1^{2}\right] ;\left[f_{0}\right]=\left[21^{N-2}\right]\end{aligned}$ \\
\hline $\begin{array}{l}{[f(a)][f(a b)]} \\
{[f(a)][f(b c)]}\end{array}$ & $-\left[\frac{N(N+1)}{2} \frac{\left(\tau_{a b}+1\right)}{\left(\tau_{a b}-1\right)}\right]^{1 / 2} \frac{1}{\prod_{a}}$ \\
\hline
\end{tabular}

$\frac{[f(a)][f(b c)]}{\left[f_{1}^{\prime \prime}\right]\left[f_{2}^{\prime \prime}\right]} \quad$ 13. $\left[f_{1}^{\prime *}\right]\left[f_{1}\right]=\left[1^{N-1}\right][1] ;\left[f_{2}^{\prime *}\right]\left[f_{2}\right]$ $=\left[1^{N-2}\right][2] ;\left[f_{0}\right]=\left[21^{N-2}\right]$

$[f(a)][f(a b)] \quad\left[\frac{N(N-1)}{2} \frac{\left(\tau_{a b}+1\right)}{\left(\tau_{a b}-1\right)}\right]^{1 / 2} \frac{1}{\Pi_{a}}$

$[f(a)][f(b c)]$

[f $\left.f_{1}^{\prime \prime}\right]\left[f_{2}^{\prime \prime}\right] \quad$ 14. $\left[f_{1}^{\prime *}\right]\left[f_{1}\right]=\left[1^{N-2}\right]\left[1^{2}\right] ;\left[f_{2}^{*}\right]\left[f_{2}\right]$

\begin{tabular}{ll}
\multicolumn{1}{c}{$=\left[2^{N-1}\right][2] ;\left[f_{0}\right]=\left[21^{N-2}\right]$} \\
\hline$[f(a b)][f(a b)]$ & $-\left[\frac{N}{2} \frac{(N+1)(N-1)}{(N+2)(N-2)}\right]^{1 / 2}\left\{\left(1+\frac{1}{\tau_{a b}}\right) \frac{1}{\Pi_{a}}\right.$ \\
& $\left.+\left(1-\frac{1}{\tau_{a b}}\right) \frac{1}{\Pi_{b}}-\frac{4}{N}\right\}$ \\
{$[f(a b)][f(a c)]$} & $-\frac{N}{2}\left[\frac{(N+1)(N-1)}{(N+2)(N-2)}\right]^{1 / 2}\left\{\left(1+\frac{1}{\tau_{a c}}\right) \frac{1}{\Pi_{a}}-\frac{4}{N}\right\}$ \\
{$[f(a b)[f(a a)]$} & $-N\left[\frac{(N+1)(N-1)}{(N+2)(N-2)}\right]^{1 / 2}\left\{\frac{1}{\Pi_{a}}-\frac{2}{N}\right\}$ \\
{$[f(a b)]$ or $[f(c c)]$} & $2\left[\frac{(N+1)(N-1)}{(N+2)(N-2)}\right]^{1 / 2}$
\end{tabular}


Table I (continued)

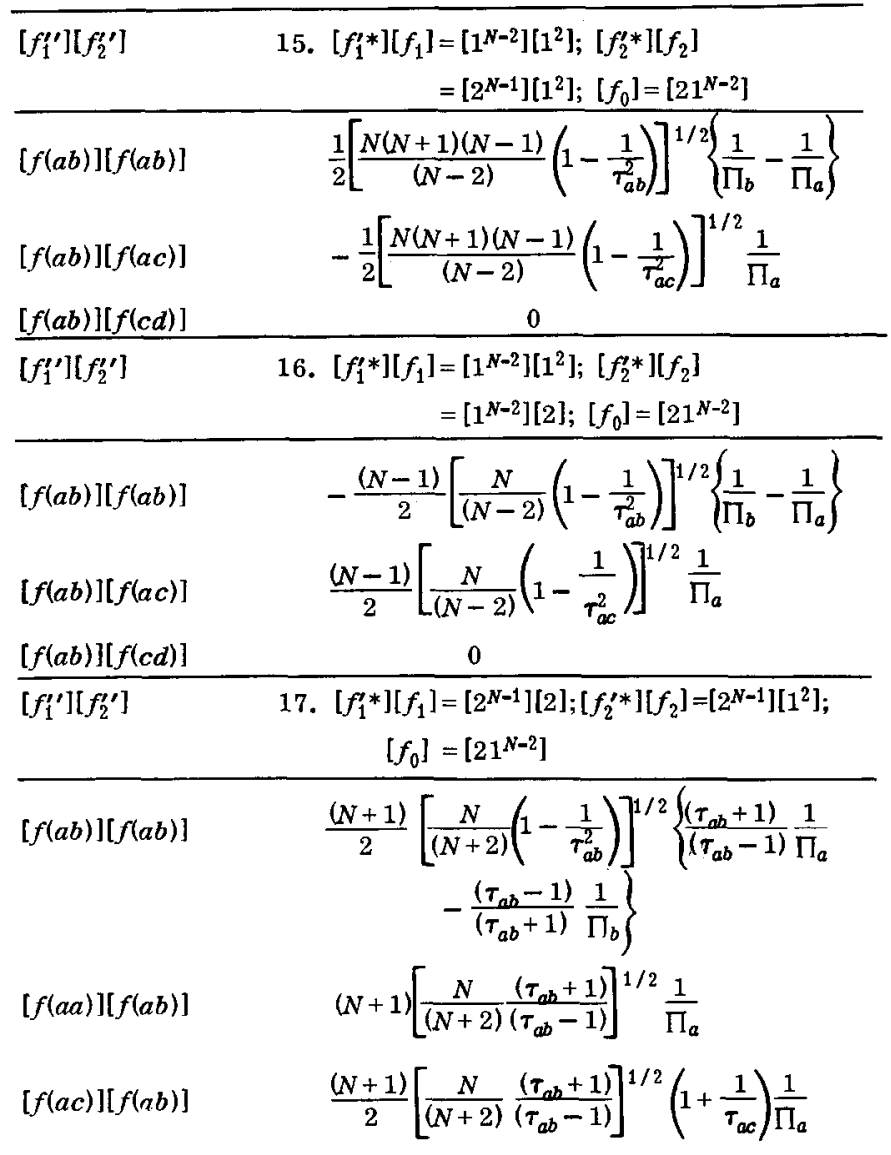

Table I (continued)

\begin{tabular}{|c|c|}
\hline $\begin{array}{l}{[f(c c)]} \\
\text { or }[f(c d)][f(a b)]\end{array}$ & 0 \\
\hline$\left[f_{1}^{\prime \prime}\right]\left[f_{2}^{\prime \prime}\right]$ & 18. $\begin{aligned} {\left[f_{1}^{\prime *}\right]\left[f_{1}\right] } & =\left[2^{N-1}\right][2] ;\left[f_{2}^{* *}\right]\left[f_{2}\right] \\
& =\left[1^{N-2}\right][2] ;\left[f_{0}\right]=\left[21^{N-2}\right]\end{aligned}$ \\
\hline$[f(a b)][f(a b)]$ & $\begin{array}{c}\frac{1}{2}\left[\frac{N(N+1)(N-1)}{(N+2)}\left(1-\frac{1}{\tau_{a b}^{2}}\right)\right]^{1 / 2}\left\{\frac{\left(\tau_{a b}-1\right)}{\left(\tau_{a b}+1\right)} \frac{1}{\Pi_{b}}\right. \\
\left.-\frac{\left(\tau_{a b}+1\right)}{\left(\tau_{a b}-1\right)} \frac{1}{\Pi_{a}}\right\}\end{array}$ \\
\hline$[f(a a)][f(a b)]$ & $-\left[\frac{N(N+1)(N-1)}{(N+2)} \frac{\left(\tau_{a b}+1\right)}{\left(\tau_{a b}-1\right)}\right]^{1 / 2} \frac{1}{\Pi_{a}}$ \\
\hline$[f(a c)][f(a b)]$ & $\begin{array}{l}-\frac{1}{2}\left[\frac{N(N+1)(N-1)}{(N+2)} \frac{\left(\tau_{a b}+1\right)}{\left(\tau_{a b}-1\right)}\right]^{1 / 2} \\
\times\left(1+\frac{1}{\tau_{a c}}\right) \frac{1}{\Pi_{a}}\end{array}$ \\
\hline $\begin{aligned} & {[f(c c)] } \\
\text { or }[f(c d)] & {[f(a b)] }\end{aligned}$ & 0 \\
\hline$\left[f_{1}^{\prime}\right]\left[f_{2}^{\prime \prime}\right]$ & 19. $\begin{aligned} {\left[f_{1}^{*}\right]\left[f_{1}\right] } & =\left[2^{N-1}\right]\left[1^{2}\right] ;\left[f_{2}^{*}\right]\left[f_{2}\right] \\
& =\left[1^{N-2}\right][2] ;\left[f_{0}\right]=\left[21^{N-2}\right]\end{aligned}$ \\
\hline$[f(a b)][f(a b)]$ & $\begin{aligned}- & \frac{1}{2}[(N+1)(N-1)]^{1 / 2}\left\{\left(1+\frac{1}{\tau_{a b}}\right) \frac{1}{\Pi_{a}}\right. \\
& \left.+\left(1-\frac{1}{\tau_{a b}}\right) \frac{1}{\Pi_{b}}\right\}\end{aligned}$ \\
\hline$[f(a c)][f(a b)]$ & $-\frac{1}{2}\left[(N+1)(N-1) \frac{\left(\tau_{a b}+1\right)}{\left(\tau_{a b}-1\right)}\left(1-\frac{1}{\tau_{a c}^{2}}\right)\right]^{1 / 2} \frac{1}{\Pi_{a}}$ \\
\hline$[f(c d)][f(a b)]$ & 0 \\
\hline
\end{tabular}

* Supported by U.S. National Science Foundation.

${ }^{1}$ J. D. Louck and L. C. Biedenharn, J. Math. Phys. 11, 2368 (1970).

${ }^{2}$ L. C. Biedenharn, J. D. Louck, E. Chacon, and M. Ciftan, J. Math. Phys. 13, 1957 (1972).

${ }^{3}$ L. C. Biedenharn and J. D. Louck, J. Math. Phys. 13, 1985 (1972).

${ }^{4}$ L. C. Biedenharn and J. D. Louck, Commun. Math. Phys. 8, 89 (1968).

${ }^{5}$ E. Chacón, M. Ciftan, and L. C. Biedenharm, J. Math. Phys. 13, 577 (1972).

${ }^{6} \mathrm{~J}$. D. Louck, Am. J. Phys. 38, 3 (1970), presents a bibliography of earlier references.

${ }^{7} J$. P. Draayer and Y. Akiyama, J. Math. Phys. 14, 1904 (1973).

${ }^{8}$ Y. Akiyama and J.P. Draayer, Comput. Phys. Commun. 5, 405 (1973).
${ }^{9} \mathrm{M}$. Moshinsky and E. Chacon, in Spectroscopic and Group Theoretical Methods in Phyics, edited by F. Bloch, et al. (North-Holland, Amsterdam, 1968).

${ }^{10}$ S.J. Alisauskas, A.-A.A. Jucys, and A. P. Jucys, J. Math. Phys. 13, 1329 (1972).

${ }^{11}$ K. T. Hecht and J. P. Draayer, Nucl. Phys. A 223, 285 (1974).

${ }^{12}$ F. S. Chang, J. B. French, and T.H. Thio, Ann. Phys. (N.Y.) 66,137 (1971).

${ }^{13}$ M. Hamermesh, Group Theory and Its Application to Physical Problems (Addison-Wesley, Reading, Mass., 1962), Chap. 7.

${ }^{14}$ I. G. Kaplan, Zh. Eksp. Teor. Fiz. 41, 560, 790 (1961) [Sov. Phys. JETP 14, 401, 568 (1962)].

${ }^{15}$ Hisashi Horie, J. Phys. Soc. Japan 19, 1783 (1964).

${ }^{16}$ D. E. Rutherford, Substitutional Analysis (Edinburgh, U. P., Edinburgh, 1948). 